\title{
Implementation of an infection prevention bundle and increased physician awareness improves surgical outcomes and reduces costs associated with spine surgery
}

\author{
*Nitin Agarwal, MD, ${ }^{1}$ Prateek Agarwal, AB, ${ }^{2}$ Ashley Querry, BS, ${ }^{1}$ Anna Mazurkiewicz, BS, ${ }^{3}$ \\ Zachary J. Tempel, MD, ${ }^{1}$ Robert M. Friedlander, MD, MA, ${ }^{1}$ Peter C. Gerszten, MD, MPH, ${ }^{1}$ \\ D. Kojo Hamilton, MD, ${ }^{1}$ David O. Okonkwo, MD, PhD, ${ }^{1}$ and Adam S. Kanter, MD'1
}

Departments of ${ }^{1}$ Neurological Surgery and ${ }^{3}$ Finance, University of Pittsburgh Medical Center, Pittsburgh; and ${ }^{2}$ Perelman School of Medicine, University of Pennsylvania, Philadelphia, Pennsylvania

\begin{abstract}
OBJECTIVE Previous studies have demonstrated the efficacy of infection prevention protocols in reducing infection rates. This study investigated the effects of the development and implementation of an infection prevention protocol that was augmented by increased physician awareness of spinal fusion surgical site infection (SSI) rates and resultant cost savings.

METHODS A cohort clinical investigation over a 10-year period was performed at a single tertiary spine care academic institution. Preoperative infection control measures (chlorohexidine gluconate bathing, Staphylococcus aureus nasal screening and decolonization) followed by postoperative infection control measures (surgical dressing care) were implemented. After the implementation of these infection control measures, an awareness intervention was instituted in which all attending and resident neurosurgeons were informed of their individual, independently adjudicated spinal fusion surgery infection rates and rankings among their peers. During the course of these interventions, the overall infection rate was tracked as well as the rates for those neurosurgeons who complied with the preoperative and postoperative infection control measures (protocol group) and those who did not (control group).

RESULTS With the implementation of postoperative surgical dressing infection control measures and physician awareness, the postoperative spine surgery infection rate decreased by $45 \%$ from $3.8 \%$ to $2.1 \%$ (risk ratio $0.55 ; 95 \% \mathrm{Cl} 0.32$ $0.93 ; p=0.03$ ) for those in the protocol cohort, resulting in an estimated annual cost savings of $\$ 291,000$. This reduction in infection rate was not observed for neurosurgeons in the control group, although the overall infection rate among all neurosurgeons decreased by $54 \%$ from $3.3 \%$ to $1.5 \%$ (risk ratio $0.46 ; 95 \% \mathrm{Cl} 0.28-0.73 ; p=0.0013$ ).

CONCLUSIONS A novel paradigm for spine surgery infection control combined with physician awareness methods resulted in significantly decreased SSI rates and an associated cost reduction. Thus, information sharing and physician engagement as a supplement to formal infection control measures result in improvements in surgical outcomes and costs.
\end{abstract}

https://thejns.org/doi/abs/10.3171/2017.11.SPINE17436

KEYWORDS physician awareness; cost consciousness; surgical site infection rates; spinal fusion surgery

$\mathrm{S}$ URGICAL site infections (SSIs) remain a prevalent postoperative complication in the United States and around the world, occurring in approximately 300,000 patients annually in the United States alone. SSIs cause a significant health care burden from both a patient and provider standpoint, as they often require reoperations and increased hospital stay. They are also associated with substantial costs and financial burden on patients and the health care system, with estimates of costs due to SSIs ranging from $\$ 3$ to $\$ 10$ billion annually. ${ }^{25}$ Given that United States health care spending is now over $17 \%$ of the gross domestic product (GDP), it is imperative that physicians develop and implement methods to reduce the rates of SSIs and associated costs.

ABBREVIATIONS $\mathrm{CHG}=$ chlorhexidine gluconate; $\mathrm{SCIP}=$ Surgical Care Improvement Project; $\mathrm{SSI}=$ surgical site infection . SUBMITTED April 10, 2017. ACCEPTED November 21, 2017.

${ }^{*}$ N.A. and P.A. contributed equally to this work. 
Much of the literature investigating the implementation of infection prevention bundles to reduce SSIs has focused on the orthopedic patient population or a heterogeneous surgical patient population. In these surgical patient populations, there is strong evidence that preoperative nasal decolonization with antibiotics (e.g., mupirocin) decreases the rate of SSIs. ${ }^{2,11,17,24}$ Furthermore, there is robust evidence that preoperative chlorhexidine gluconate (CHG) bathing also decreases the rate of SSIs in these surgical patient populations. ${ }^{15,28}$

SSIs are a known potential complication following spine surgery, particularly when instrumentation is employed. Most studies report the incidence of SSIs following spine surgery to be anywhere between $0.5 \%$ and $5 \% .^{12,13,16,19}$ However, there exists a lack of literature on the implementation of infection prevention bundles to reduce SSIs specifically in patients undergoing spine surgery, although there has been discussion of the potential benefits of such bundles in spine surgery patients. ${ }^{6,29}$

Besides formal infection prevention bundles, the literature has not adequately explored the effect of physician awareness of interventions on health care risks such as SSIs. It is plausible that such physician awareness interventions are an effective adjunct to mandatory interventions to reduce health care treatment risks, particularly given the demonstrated effect of physician awareness with regards to prescription drug and diagnostic test costs. This is further supported by the notion that surgeons may often not be aware of their own, independently adjudicated postoperative infection rates. ${ }^{1,10,21,23}$

The purpose of this study was to specifically examine the effects of implementation of an infection prevention bundle and at the same time an increased physician awareness to improve postoperative outcomes and reduce costs for spinal fusion surgery.

\section{Methods}

Within the department of neurological surgery at a single institution, Surgical Care Improvement Project (SCIP) measures (prophylactic antibiotic, appropriate hair removal) had been in place since 2004, with documentation of infection rates relating to spinal fusion surgery since 2007.

In 2011, a multispecialty committee recommended the following preoperative spinal fusion surgery infection control measures to physicians: 1) $4 \%$ CHG preoperative bathing for 5 days $(\$ 3.31$ per bottle; Exidine Scrub Solution 4\%; Becton, Dickinson and Company), 2) nasal screening for Staphylococcus aureus preoperatively with administration of $2 \%$ mupirocin ointment for nasal decolonization for 5 days for positive tests (approximately \$20), 9,11 and 3) CHG-alcohol as the standard preoperative preparation unless contraindicated.

Once the preoperative bundle was firmly in place and physicians were comfortable with it, in 2013, the following additional postoperative measures were recommended: 1 ) requiring sterile technique for surgical dressing changes, 2) requiring dressings to be changed daily for 7 days after spine surgery, and 3) standardization of dressing changes.

Besides overall infection rate tracking, infection rates were tracked for individual neurosurgeons who complied with these measures (protocol) as well as neurosurgeons who did not comply with these measures (control).

Physician awareness was also implemented in 2015 in which all attending neurosurgeons (both protocol and control groups) and residents were informed of their individual infection rates for spinal fusion surgeries and their infection rate ranking when compared with their colleagues. These initiatives were discussed in both resident and faculty departmental quality improvement conferences. Infections were independently adjudicated by infection control staff for individual residents and faculty based on the Centers for Disease Control and Prevention's National Healthcare Safety Network definitions of an SSI.

\section{Results \\ Overall Infection Rates}

From January 2007 to February 2011, the baseline infection rate for spinal fusion surgery in the department of neurological surgery among all neurosurgeons averaged 1.3\% (111 infections/8751 procedures). The overall spinal fusion infection rate had also been steadily rising over time, with an annual rate of $0.8 \%$ in $2007,1.2 \%$ in 2008 , $1.4 \%$ in $2009,1.7 \%$ in 2010 , and $2.9 \%$ in 2011.

In an attempt to combat this rise in neurosurgical spinal fusion infection rates, spine infection control measures were begun in March 2011 that included 4\% CHG preoperative bathing, nasal screening for and decolonization of $S$. aureus, and CHG-alcohol for preoperative preparation. However, despite these measures, the average spine surgery infection rate from March 2011 to December 2012 for all neurosurgeons increased to $3.3 \%$ (69 infections/2108 procedures) (risk ratio 2.58; 95\% CI 1.92-3.47; $\mathrm{p}<0.0001$ ). At this time, surgeon compliance with the bundle was less than $50 \%$, and several measures were implemented to increase compliance.

Consequently, in January 2013, additional spine infection control measures were recommended, including sterile technique for surgical dressing changes, dressings to be changed daily for 7 days postoperatively, and standardized dressing changes. The infection rate declined from 3.3\% to an average of 2.3\% from January 2013 to April 2015 (108 infections/4676 procedures) (risk ratio $0.71 ; 95 \%$ CI $0.52-0.95 ; \mathrm{p}=0.03$ ).

Finally, a physician awareness program was implemented in May 2015 in which all attending neurosurgeons and residents were individually ranked based upon their infection rates and received an email copied to the department chair of any newly occurring infections. The infection rate trended downward from $2.3 \%$ to an average of $1.5 \%$ between May 2015 and July 2016 (22 infections/1474 procedures) (risk ratio $0.65 ; 95 \% \mathrm{CI} 0.41-1.02 ; \mathrm{p}=0.07$ ) (Fig. 1).

While the $1.3 \%$ baseline overall spine surgery infection rate was not significantly different from the $1.5 \%$ average infection rate from May 2015 to July 2016 (risk ratio 1.18 ; 95\% CI $0.75-1.85 ; \mathrm{p}=0.57$ ), there was a significant $54 \%$ decline in the infection rate from when preoperative infection control measures were recommended (3.3\%; March 2011 to December 2012) to when both postoperative dressing and physician awareness were also in place 


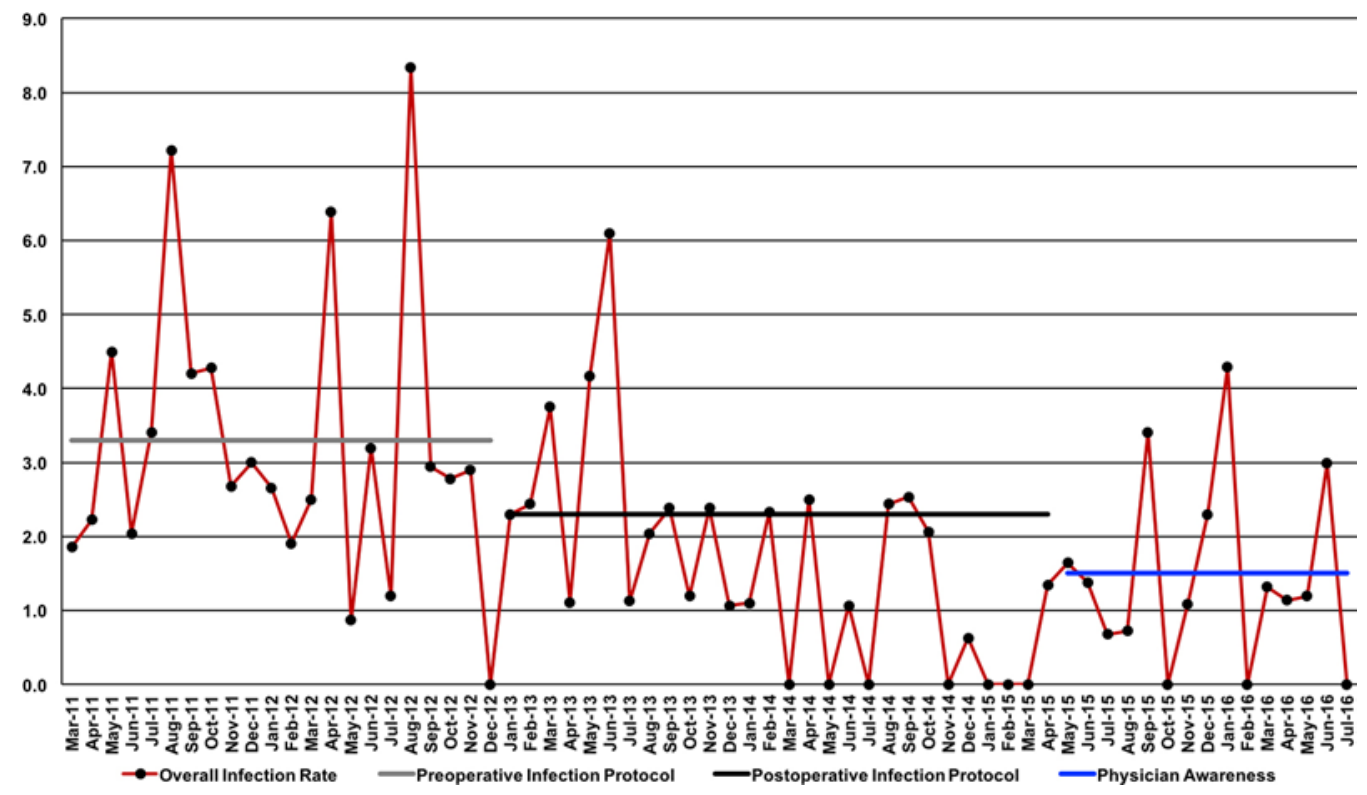

FIG. 1. Overall spinal fusion postoperative SSI rate, including neurosurgeons in both the protocol and control cohorts, depicting a significant decrease in the infection rate after the implementation of a postoperative infection control protocol (surgical dressing measures) in January 2013 and physician awareness in May 2015. Figure is available in color online only.

(1.5\%; May 2015 to July 2016) (risk ratio $0.46 ; 95 \%$ CI $0.28-0.73 ; \mathrm{p}=0.0013)$.

\section{Protocol Cohort}

In addition to observing the overall spinal fusion surgery infection rate, an aim of the investigation was to determine the infection rate for neurosurgeons in the protocol versus those in the control cohort, namely those who complied with the infection prevention bundle and those who did not.

From January 2007 to February 2011, the baseline infection rate for protocol participants averaged 3.0\% (64 infections/2108 procedures). When preoperative infection control measures were recommended, the infection rate for neurosurgeons in the protocol cohort trended upward to $3.8 \%$ from March 2011 to December 2012 (61 infections/1622 procedures) (risk ratio 1.24; 95\% CI 0.88-1.75; $\mathrm{p}=0.26$ ).

After postoperative surgical dressing measures were recommended, the average infection rate trended downward from 3.8\% to an average of 2.9\% from January 2013 to April 2015 (93 infections/3260 procedures) (risk ratio $0.76 ; 95 \%$ CI $0.55-1.04 ; \mathrm{p}=0.1$ ) When a physician awareness program was implemented, the infection rate further trended downward from $2.9 \%$ to an average of $2.1 \%$ from May 2015 to July 2016 (17 infections/829 procedures) (risk ratio 0.72 ; 95\% CI $0.43-1.2 ; \mathrm{p}=0.25$ ) (Fig. 2).

While the $2.1 \%$ average infection rate from May 2015 to July 2016 was only slightly lower than the $3.0 \%$ baseline spine surgery infection rate for those in the protocol (risk ratio $0.68 ; 95 \%$ CI $0.40-1.12 ; \mathrm{p}=0.18$ ), there was a significant $45 \%$ decline in the infection rate from when preoperative infection control measures were recommended (3.8\%; March 2011 to December 2012) to when both post- operative surgical dressing measures and individual physician awareness where also in place (2.1\%; May 2015 to July 2016) (risk ratio 0.55 ; 95\% CI 0.32-0.93; $\mathrm{p}=0.03$ ).

\section{Control Cohort}

From January 2007 to February 2011, the baseline infection rate for those in the control cohort averaged $0.7 \%$ (47 infections/6643 procedures). Subsequently, preoperative and postoperative infection control measures were recommended within the department, with which the control group did not comply. When preoperative infection control measures were recommended, the infection rate for the control cohort increased to 1.6\% from March 2011 to December 2012 (8 infections/486 procedures) (risk ratio $2.33 ; 95 \%$ CI $1.1-4.9 ; \mathrm{p}<0.05)$.

After postoperative surgical dressing measures were recommended, the average infection rate decreased from $1.6 \%$ to an average of $1.1 \%$ from January 2013 to April 2015 (15 infections/1416 procedures) (risk ratio 0.64; $95 \%$ CI $0.27-1.5 ; \mathrm{p}=0.43$ ). When physician awareness was added, the infection rate again marginally decreased from $1.1 \%$ to an average of $0.8 \%$ from May 2015 to July 2016 (5 infections $/ 645$ procedures) (risk ratio $0.73 ; 95 \%$ CI $0.27-$ $2.0 ; p=0.71$ ) (Fig. 3).

While the $0.7 \%$ baseline spine surgery infection rate for the control group was not significantly different from the $0.8 \%$ average infection rate from May 2015 to July 2016 (risk ratio 1.08; 95\% CI 0.43-2.7; p > 0.5), there was a downward trend in infection rate from when preoperative infection control measures were recommended (1.6\%; March 2011 to December 2012) to when both postoperative surgical dressing measures and physician awareness were also in place (0.8\%; May 2015 to July 2016) (risk ratio $0.47 ; 95 \%$ CI $0.16-1.43 ; \mathrm{p}=0.28$ ). 


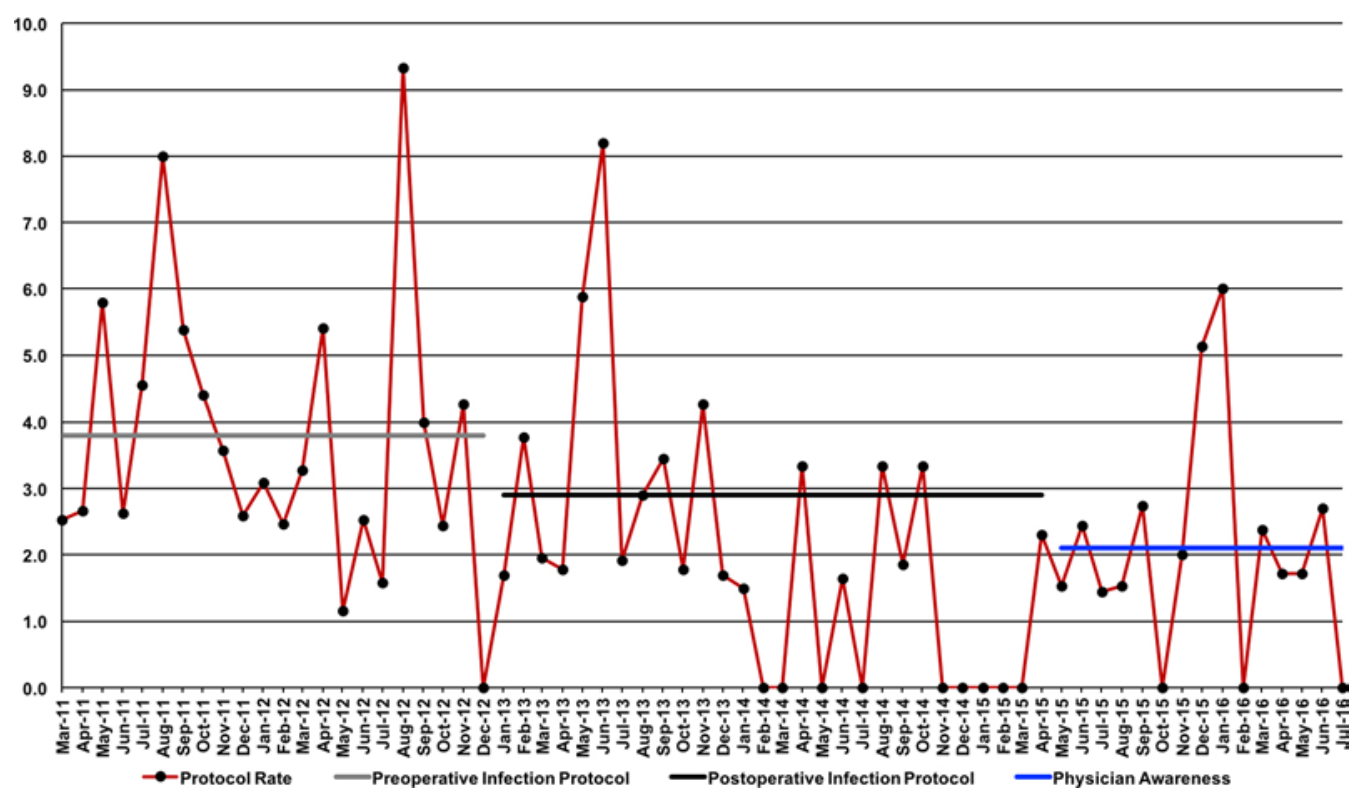

FIG. 2. Spinal fusion postoperative SSI rate for the protocol cohort, depicting a significant decrease in the infection rate after the implementation of a postoperative infection control protocol (surgical dressing measures) in January 2013 and physician awareness in May 2015. Figure is available in color online only.

\section{Infection Cost Savings: Protocol Cohort}

Using an average inpatient hospital cost of $\$ 19,400$ for treating postoperative spinal fusion surgery infections, the estimated annual infection treatment cost for neurosurgeons in the protocol cohort was calculated for the baseline period (January 2007 to February 2011), the period when preoperative infection control measures were recommended and initiated (March 2011 to December 2012), and the period after both postoperative surgical dressing measures and physician awareness were implemented
(May 2015 to July 2016). In this calculation, infection and procedure numbers were annualized and the number of infections were further adjusted based upon the ratio of the annualized volume of procedures between the two periods being compared. The average inpatient hospital cost of $\$ 19,400$ was derived from collecting actual inpatient costs from patient encounters with the Division of Infectious Diseases. This average cost represents all direct and semivariable hospital costs accumulated by these patients while they were treated for infections within the hospital.

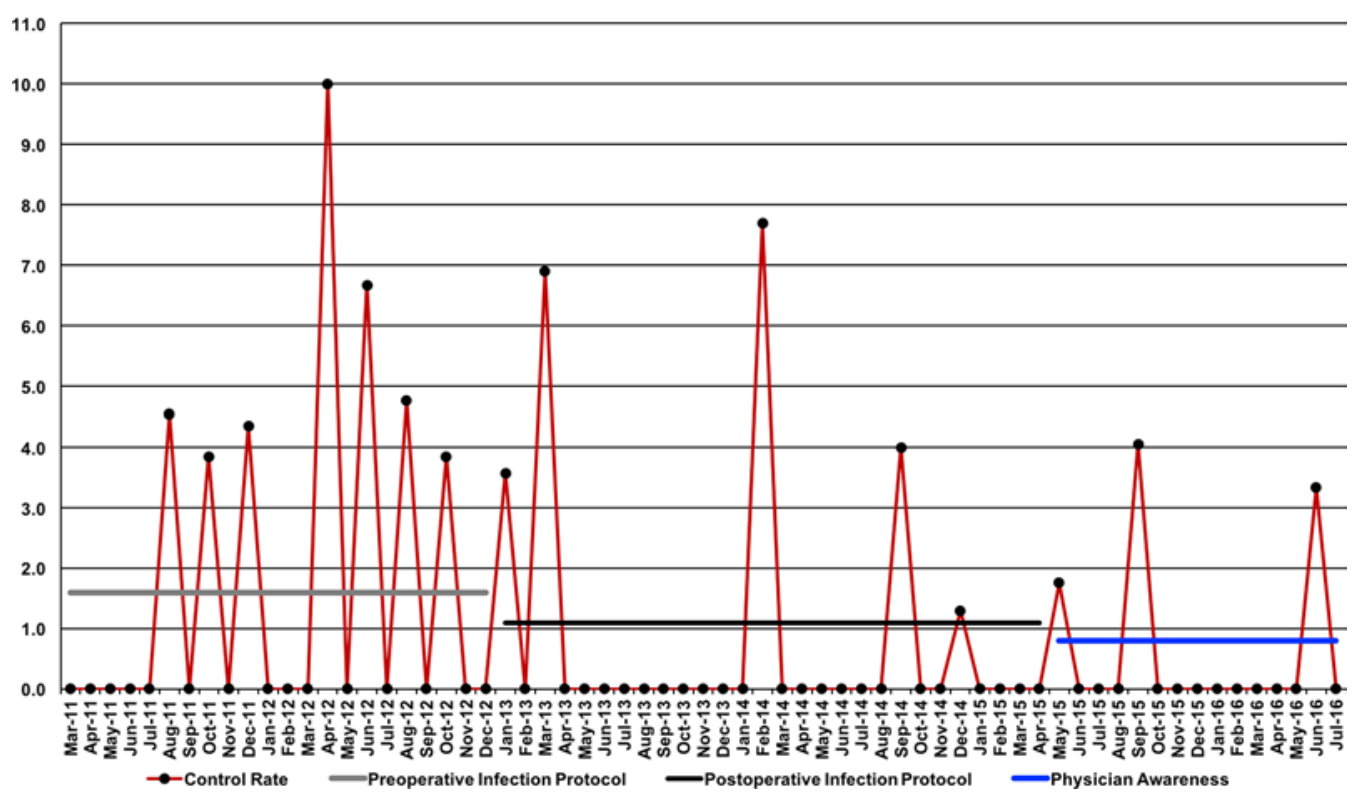

FIG. 3. Spinal fusion postoperative SSI rate for the control cohort, depicting a downward trend in the infection rate with the implementation of physician awareness in May 2015. Figure is available in color online only. 
When comparing the baseline period to the period after both postoperative surgical dressing measures and physician awareness were in place, the estimated average annual cost savings was $\$ 97,000$ from preventing postoperative spine surgery infections. When comparing the period when preoperative infection control measures were recommended to the period after both postoperative surgical dressing measures and physician awareness were in place, the estimated average annual cost savings was $\$ 291,000$ from preventing postoperative spine surgery infections. As outpatient costs were not readily accessible, this is likely an underestimate of the true total costs since it only incorporates inpatient costs. Furthermore, these are only direct costs and not indirect costs to patients and their families.

\section{Discussion}

This study presents a novel investigation within the subspecialty field of neurosurgery that examined the effects of infection prevention protocol measures as well as individual physician awareness on infection rates and related cost reduction for spinal fusion surgery. The study found mixed results with regards to the various interventions implemented over the time period examined. Considering those in the protocol cohort who complied with the infection prevention bundle, preoperative infection prevention measures did not appear to be effective, as the infection rate actually trended upward from a $3.0 \%$ baseline to $3.8 \%$ after these measures were implemented. However, infection rates did trend downward when postoperative surgical dressing was implemented, from $3.8 \%$ to $2.9 \%$, and then trended downward further to $2.1 \%$ after a physician awareness program was initiated. This resulted in a statistically significant $45 \%$ overall reduction in the infection rate following the implementation of postoperative surgical dressing measures and physician awareness (3.8\% to $2.1 \%$ ). Moreover, an associated annual inpatient cost savings of $\$ 291,000$ was noted. This same statistically significant reduction in infection rates as a result of postoperative surgical dressing measures and physician awareness was not observed for neurosurgeons in the control cohort but was observed for the overall infection rate (3.3\% to $1.5 \%)$.

\section{Infection Control Protocols}

Within the realm of spine surgery, there is a paucity of literature on the implementation of infection prevention bundles to decrease the rate of postoperative infection. Most of the literature that does exist revolves around the use of intraoperative vancomycin or other specific individual measures to reduce the risk of SSI. While some of these studies have found a benefit to using intraoperative vancomycin during spine surgery, ${ }^{3,4}$ others have found no difference in the incidence of SSI between patients who receive intraoperative vancomycin and those who do not. ${ }^{20,27}$ Moreover, there is some evidence suggesting that the use of intraoperative vancomycin is associated with increased complications, which further brings into question the benefits of topical vancomycin as a tool to reduce the rate of SSIs in spine surgery. ${ }^{8,14}$

Considering that the benefits of vancomycin in prevent- ing SSIs are unclear in spine surgery, it is only reasonable to investigate the use of infection prevention bundles as a method to reduce the rate of SSIs, which has been performed in the present study. In surgical patient populations other than spine surgery, randomized controlled trials already exist that demonstrate the ability of preoperative nasal decolonization with antibiotics and CHG bathing to reduce the rate of SSIs. ${ }^{2,11,28}$ One recent study did report the use of an infection prevention bundle to reduce SSIs in spine surgery. In this study, Featherall et al. found that an infection prevention bundle reduced the rate of postoperative infection in spine surgery by $52 \%$, from $4.12 \%$ to $2.0 \%$, with an average infection treatment cost of $\$ 25,962$.

Interestingly, the results of the present study somewhat contradict those of the Featherall et al. study in that we did not observe a reduction in infection rate following the implementation of preoperative prevention measures, including nasal decolonization with antibiotics and CHG bathing. In fact, we actually observed an upward trend in the infection rate for neurosurgeons in the protocol cohort and a significant increase in the overall infection rate following the implementation of these measures. However, unlike the Featherall et al. study, we subsequently included standardization of dressing changes and required dressing to be changed daily for 7 days following spine surgery. These postoperative surgical dressing measures were associated with a downward trend in the infection rate for neurosurgeons in the protocol cohort and significantly decreased the overall infection rate, suggesting that postoperative surgical dressing measures may be more effective in preventing SSI than preoperative nasal decolonization/ CHG bathing in spine surgery.

Another potential explanation for the difference in our results compared with those of the Featherall et al. study relates to compliance rates. When the preoperative infection bundle was implemented from March 2011 to December 2012, compliance was less than 50\%, which could partially explain the initial rise in infection rates. Given the predominance of evidence demonstrating the ability of preoperative infection control measures to reduce the rate of SSIs in other surgical specialties, we continued with the preoperative bundle and added a postoperative bundle and physician awareness program as well. Compliance was higher during these time periods from January 2013 to April 2015 (postoperative bundle added) and from May 2015 to July 2016 (physician awareness added). Ultimately, this increase in compliance resulted in a statistically significant reduction in the infection rate following the implementation of postoperative surgical dressing measures and physician awareness for protocol cohort.

Despite this increase in compliance, one might still expect the infection rate from March 2011 to December 2012 to have at least remained the same, not increased, with the implementation of preoperative infection control measures. However, it is important to note that the annual overall infection rate was steadily rising during the baseline period from $0.8 \%$ in 2007 to $2.9 \%$ in 2011, which provided the initial impetus for implementation of preoperative infection control measures. As such, perhaps this first set of preoperative infection control measures may not have been sufficient to halt the increase in infection rates. 


\section{Infection Prevention Cost Savings}

The associated annual inpatient cost savings from reducing the rate of infections was calculated using an average inpatient hospital cost of $\$ 19,400$, which was derived from actual inpatient costs from patient encounters with the Infectious Diseases service. Although our calculations of cost savings are simply estimates, the average inpatient hospital cost of treating infection is similar to the Featherall et al. reported average infection treatment cost of $\$ 25,962$. We also ensured to control for number of months and case volume during a time period by annualizing infection and procedure numbers and further adjusting the number of infections based on the ratio of the annualized volume of procedures between the two periods being compared.

\section{Infection Control via Physician Awareness}

In this study, a physician awareness intervention program was initiated in which individual neurosurgeons were informed of personal infection rates for spine surgeries and their infection rate ranking was compared with that of their colleagues. All initiatives were discussed in both resident and faculty departmental quality improvement conferences. Through effective transmission of knowledge, facilitation of reflective practice through feedback, and establishment of a supportive environment, we believe that physician awareness contributed to the reduction of postoperative infection rates and associated costs. ${ }^{26}$ We observed a statistically significant decline in the infection rate for protocol cohort from when only preoperative infection prevention measures were in place to when both postoperative surgical dressing measures and physician awareness were added. Thus, our results support that concomitant implementation of an infection prevention bundle and physician awareness is a most effective strategy. As for neurosurgeons in the control group, there was only a downward trend rather than a statistically significant reduction in the postoperative infection rate during the same time periods. Though this comparison was not sufficiently powered to detect a statistical difference due to the lower number of procedures and postoperative infections, it supports the idea that physician awareness alone may not be sufficient to prevent SSIs.

\section{Limitations}

The present study does have limitations, most notably that this was not a randomized controlled study. It is plausible that factors other than the infection prevention bundle implemented could be truly responsible for the reduction in postoperative infection rates that were observed. Although we do not believe there were significant differences in case complexity or patient comorbidities over time, our study does not account for the possibility that simpler operations and healthier patients could be driving the reduction in postoperative infection rates. Even if there was no change in case complexity or patient comorbidities over time, an increase in minimally invasive surgeries over time could be responsible for the decrease in infection rates, as these surgeries have been shown to be associated with lower postoperative infection rates in the literature. ${ }^{5,18,22}$ Anoth- er potential confounding factor in this study is the use of intraoperative vancomycin powder for postoperative SSI prophylaxis. While some neurosurgeons in our department do use intraoperative vancomycin during spine surgery, it is not a mandated intervention or routinely used. Therefore, we did not investigate the effect of vancomycin powder usage on infection rates in the present study.

We must also acknowledge that possible confounding factors such as the ones discussed above are also a limitation when comparing the protocol and control cohorts. For instance, the control cohort had a lower baseline infection rate $(0.7 \%)$ than the protocol cohort $(3.0 \%)$, which may indicate differences in case complexity, surgical approach, or patient comorbidities. These factors may have also changed differently over time in the protocol and control cohorts, which further limits comparison of the two groups.

Therefore, future randomized controlled experiments within spine surgery might be performed to control for confounding variables and determine the isolated effect of an infection prevention bundle and physician awareness on postoperative SSI rates. Future studies would also benefit from data on speciation of isolated organisms and their sensitivities to various antibiotics to better understand the specific types of bacteria that are being controlled more effectively with the proposed interventions.

\section{Conclusions}

Unique spine surgery infection control measures combined with physician awareness successfully decreased postoperative SSI, thereby also resulting in significant cost savings. Consequently, the spine surgery infection control measures presented here should be employed routinely to reduce surgical treatment risk, while physician awareness should be used as a supplement to further improve surgical safety and reduce costs.

\section{References}

1. Agarwal N, Agarwal P, Querry A, Mazurkiewicz A, Whiteside B, Marroquin OC, et al: Reducing surgical infections and implant costs via a novel paradigm of enhanced physician awareness. Neurosurgery [epub ahead of print], 2017

2. Bode LG, Kluytmans JA, Wertheim HF, Bogaers D, Vandenbroucke-Grauls CM, Roosendaal R, et al: Preventing surgical-site infections in nasal carriers of Staphylococcus aureus. N Engl J Med 362:9-17, 2010

3. Caroom C, Tullar JM, Benton EG Jr, Jones JR, Chaput CD: Intrawound vancomycin powder reduces surgical site infections in posterior cervical fusion. Spine (Phila Pa 1976) 38:1183-1187, 2013

4. Dennis HH, Wei DT, Darren KZ, Shantakumar JT, Kumar $\mathrm{N}$, Lau LL, et al: Is intraoperative local vancomycin powder the answer to surgical site infections in spine surgery? Spine (Phila Pa 1976) 42:267-274, 2017, 2016

5. Ee WW, Lau WL, Yeo W, Von Bing Y, Yue WM: Does minimally invasive surgery have a lower risk of surgical site infections compared with open spinal surgery? Clin Orthop Relat Res 472:1718-1724, 2014

6. Epstein NE: Preoperative, intraoperative, and postoperative measures to further reduce spinal infections. Surg Neurol Int 2:17, 2011

7. Featherall J, Miller JA, Bennett EE, Lubelski D, Wang H, Khalaf T, et al: Implementation of an infection prevention 
bundle to reduce surgical site infections and cost following spine surgery. JAMA Surg 151:988-990, 2016

8. Ghobrial GM, Cadotte DW, Williams K Jr, Fehlings MG, Harrop JS: Complications from the use of intrawound vancomycin in lumbar spinal surgery: a systematic review. Neurosurg Focus 39(4):E11, 2015

9. Hacek DM, Robb WJ, Paule SM, Kudrna JC, Stamos VP, Peterson LR: Staphylococcus aureus nasal decolonization in joint replacement surgery reduces infection. Clin Orthop Relat Res 466:1349-1355, 2008

10. Hickson GB, Pichert JW, Webb LE, Gabbe SG: A complementary approach to promoting professionalism: identifying, measuring, and addressing unprofessional behaviors. Acad Med 82:1040-1048, 2007

11. Kalmeijer MD, Coertjens H, van Nieuwland-Bollen PM, Bogaers-Hofman D, de Baere GA, Stuurman A, et al: Surgical site infections in orthopedic surgery: the effect of mupirocin nasal ointment in a double-blind, randomized, placebo-controlled study. Clin Infect Dis 35:353-358, 2002

12. Kanayama M, Hashimoto T, Shigenobu K, Oha F, Togawa D: Effective prevention of surgical site infection using a Centers for Disease Control and Prevention guideline-based antimicrobial prophylaxis in lumbar spine surgery. J Neurosurg Spine 6:327-329, 2007

13. Kang BU, Lee SH, Ahn Y, Choi WC, Choi YG: Surgical site infection in spinal surgery: detection and management based on serial C-reactive protein measurements. J Neurosurg Spine 13:158-164, 2010

14. Kang DG, Holekamp TF, Wagner SC, Lehman RA Jr: Intrasite vancomycin powder for the prevention of surgical site infection in spine surgery: a systematic literature review. Spine J 15:762-770, 2015

15. Kapadia BH, Jauregui JJ, Murray DP, Mont MA: Does preadmission cutaneous chlorhexidine preparation reduce surgical site infections after total hip arthroplasty? Clin Orthop Relat Res 474:1583-1588, 2016

16. Klemencsics I, Lazary A, Szoverfi Z, Bozsodi A, Eltes P, Varga PP: Risk factors for surgical site infection in elective routine degenerative lumbar surgeries. Spine J 16:1377-1383, 2016

17. Kluytmans J, Harbarth S: Methicillin-resistant Staphylococcus aureus decolonization: "yes, we can," but will it help? Infect Control Hosp Epidemiol 30:633-635, 2009

18. Kulkarni AG, Patel RS, Dutta S: Does minimally invasive spine surgery minimize surgical site infections? Asian Spine J 10:1000-1006, 2016

19. Lewkonia P, DiPaola C, Street J: Incidence and risk of delayed surgical site infection following instrumented lumbar spine fusion. J Clin Neurosci 23:76-80, 2016

20. Martin JR, Adogwa O, Brown CR, Kuchibhatla M, Bagley CA, Lad SP, et al: Experience with intrawound vancomycin powder for posterior cervical fusion surgery. J Neurosurg Spine 22:26-33, 2015

21. Martinez W, Hickson GB, Miller BM, Doukas DJ, Buckley JD, Song J, et al: Role-modeling and medical error disclosure: a national survey of trainees. Acad Med 89:482-489, 2014

22. O'Toole JE, Eichholz KM, Fessler RG: Surgical site infection rates after minimally invasive spinal surgery. J Neurosurg Spine 11:471-476, 2009

23. Pichert JW, Hickson G, Moore I: Using patient complaints to promote patient safety, in Henriksen K, Battles JB, Keyes MA, et al (eds): Advances in Patient Safety: New Directions and Alternative Approaches (Vol. 2: Culture and Redesign). Rockville: Agency for Healthcare Research and Quality, 2008

24. Schweizer ML, Chiang HY, Septimus E, Moody J, Braun B, Hafner J, et al: Association of a bundled intervention with surgical site infections among patients undergoing cardiac, hip, or knee surgery. JAMA 313:2162-2171, 2015

25. Scott RD II: The Direct Medical Costs of HealthcareAssociated Infections in U.S. Hospitals and the Benefits of Prevention. Atlanta: Centers for Disease Control and Prevention, 2009

26. Stammen LA, Stalmeijer RE, Paternotte E, Oudkerk Pool A, Driessen EW, Scheele F, et al: Training physicians to provide high-value, cost-conscious care: a systematic review. JAMA 314:2384-2400, 2015

27. Suh BK, Moon SH, Kim TH, Oh JK, Kwon YS, Park JS, et al: Efficacy of antibiotics sprayed into surgical site for prevention of the contamination in the spinal surgery. Asian Spine J 9:517-521, 2015

28. Swan JT, Ashton CM, Bui LN, Pham VP, Shirkey BA, Blackshear JE, et al: Effect of chlorhexidine bathing every other day on prevention of hospital-acquired infections in the surgical ICU: a single-center, randomized controlled trial. Crit Care Med 44:1822-1832, 2016

29. Thakkar V, Ghobrial GM, Maulucci CM, Singhal S, Prasad SK, Harrop JS, et al: Nasal MRSA colonization: impact on surgical site infection following spine surgery. Clin Neurol Neurosurg 125:94-97, 2014

\section{Disclosures}

A.S.K. is a consultant for NuVasive. He is a patent holder and receives royalties from Zimmer Biomet.

\section{Author Contributions}

Conception and design: N Agarwal, Friedlander. Acquisition of data: N Agarwal, Querry, Mazurkiewicz. Analysis and interpretation of data: N Agarwal, P Agarwal, Querry, Mazurkiewicz. Drafting the article: N Agarwal, P Agarwal, Querry, Mazurkiewicz. Critically revising the article: Kanter, N Agarwal, P Agarwal, Tempel, Friedlander, Gerszten, Hamilton, Okonkwo. Reviewed submitted version of manuscript: all authors. Approved the final version of the manuscript on behalf of all authors: Kanter. Statistical analysis: P Agarwal. Administrative/ technical/material support: Kanter, Friedlander. Study supervision: Kanter.

\section{Supplemental Information}

\section{Previous Presentations}

Spine Summit 2017-33rd Annual Meeting of the Section on Disorders of the Spine and Peripheral Nerves, Las Vegas, NV, March 8-11, 2017.

\section{Correspondence}

Adam S. Kanter: University of Pittsburgh Medical Center, Pittsburgh,PA.kanteras@upmc.edu. 